\title{
The biochemical mechanism of caspase-2 activation
}

\author{
BC Baliga ${ }^{1}$, SH Read ${ }^{1}$ and S Kumar ${ }^{*, 1,2}$ \\ ${ }^{1}$ Hanson Institute, IMVS, Frome Road, Adelaide 5000, Australia \\ 2 Department of Medicine, Adelaide University, Adelaide 5005, Australia \\ * Corresponding author: S Kumar, Hanson Institute, PO Box 14, Rundle Mall, \\ Adelaide SA 5000, Australia. Tel: +61-8-8222-3738; \\ Fax: +61-8-8222-3139; E-mail: sharad.kumar@imvs.sa.gov.au
}

Received 06.2.04; revised 15.5.04; accepted 11.6.04; published online 06.8.04 Edited by GM Cohen

\begin{abstract}
A unified model for initiator caspase activation has previously been proposed based on the biochemical analysis of caspase-8 and -9 . Caspase-2 is structurally related to caspase-9, but its mechanism of activation is not known. Using an uncleavable mutant of caspase-2, we show that dimerization (and not processing) is the key event that drives initial procaspase-2 activation. Following dimerization, caspase-2 undergoes autocatalytic cleavage that promotes its stable dimerization and further enhances the catalytic activity of caspase-2. Although the caspase-2 zymogen does not require cleavage for the initial acquisition of activity, intersubunit cleavage is required to generate levels of activity required to induce cell death by overexpression. We also provide evidence that the reported disulfide bond linkage between two caspase-2 monomers is dispensable for caspase-2 dimerization. As caspase-2 does not require cleavage for its initial activation, our findings confirm caspase-2 to be a bona fide initiator caspase.

Cell Death and Differentiation (2004) 11, 1234-1241.

doi:10.1038/sj.cdd. 4401492

Published online 6 August 2004
\end{abstract}

Keywords: caspase-2; dimerization; activation; proteolytic cleavage; initiator caspase

Abbreviations: CARD, caspase recruitment domain; GST, glutathione $S$-transferase; PAGE, polyacrylamide gel electrophoresis; PCR, polymerase chain reaction; WT, wild type; IVT, in vitro translated

\section{Introduction}

Activation of the caspase family of cysteine proteases is a key event in the initiation and execution of apoptosis. ${ }^{1-3}$ Caspases are present as inactive zymogens in healthy cells and are grouped into two classes, based on their structural similarity. The initiator caspases (caspase-8, $-9,-10$ ) have a long aminoterminal prodomain that contains specific protein-protein interaction motifs that mediate binding to adaptor proteins. Based on extensive biochemical analysis of caspase-8 and -9 , a model for apical caspase activation has been proposed whereby autocatalytic activation occurs upon dimerization of caspase zymogens, ${ }^{4-7}$ a process likely to be facilitated by adaptor proteins in vivo. In contrast to the initiator caspases, the effector caspases (caspase-3, -6, -7) pre-exist as dimers in healthy cells. ${ }^{2}$ Effector caspases have a short prodomain and their activation requires proteolytic cleavage of the protease domain performed by activated initiator caspases. Once activated, effector caspases cleave a large number of cellular substrates resulting in the morphological changes that characterize apoptotic cell death. ${ }^{8}$

Caspase-2 has a long prodomain which contains a caspase recruitment domain (CARD), thus defining it as one of the initiator caspases. ${ }^{9-11}$ Caspase-2 activation occurs rapidly in response to diverse apoptotic signals. ${ }^{12}$ When overexpressed, caspase-2 activation occurs by an autoproteolytic mechanism, but the precise mechanism of its activation is unknown. ${ }^{13}$ The CARD plays an essential role in initial caspase-2 oligomerization/activation and the fusion of the caspase-2 prodomain to caspase-3 generates an autoactivating chimeric molecule. ${ }^{14-16}$ Caspase-2 can bind to the CARDcontaining molecule RAIDD. ${ }^{17-19}$ Recent data suggest that PIDD, a p53-induced death domain-containing protein, binds RAIDD and facilitates caspase-2 activation in response to genotoxic stress. ${ }^{20}$ Consistent with a nonredundant initiator function, caspase-2 ablation by RNAi inhibits apoptosis in various distinct contexts including, stress-induced apoptosis in some transformed cell lines, ${ }^{21}$ p53-dependent apoptosis in colorectal cells following Bcl-2 inhibition, ${ }^{22}$ cell death as a result of mitotic catastrophe, ${ }^{23}$ and apoptosis induced by the expression of NRIF3 family of transcriptional regulators in breast cancer cells. ${ }^{24}$ Similar studies employing caspase-2 antisense, inhibitors and recombinant caspase-2 have implicated a role for caspase-2 upstream of mitochondrial permeabilization. ${ }^{25-30}$

Although a CARD-mediated, adaptor-assisted, autocatalytic activation mechanism clearly indicates that caspase- 2 is an initiator caspase, there is conflicting evidence as to whether caspase-2 actually functions as an initiator caspase during apoptosis. Unlike caspase-8 and -9 , caspase-2 does not directly cleave any other mammalian caspase aside from its own precursor. ${ }^{27}$ Procaspase-2 is efficiently cleaved by recombinant caspase- 3 in vitro and cleavage of caspase-2 is detected downstream of caspase-3 activation in many experimental systems. ${ }^{31-34}$ These data have suggested that rather than initiating the caspase activation cascade in response to death signals, caspase-2 may function to amplify the signal downstream of caspase-3 activation. ${ }^{35,36}$ An amplifying function would be consistent with the lack of an overt phenotype in caspase-2 knockout mice. ${ }^{37}$

The recently described crystal structure of human caspase2 reveals that its overall fold is similar to that of other caspases for which structures have been determined previously. ${ }^{38}$ Fully processed caspase-2 bound to the Ac-LDESD-aldehyde inhibitor forms a $(\mathrm{p} 19+\mathrm{p} 12)_{2}$ dimer that contains two active sites, one in each monomer. Interestingly, unlike caspase-8 and -9 , unbound caspase- 2 is apparently a stable dimer in solution. ${ }^{38}$ Under crystallization conditions, two caspase-2 monomers are covalently linked by a central disulfide bridge, 
a unique feature of caspase-2. ${ }^{38}$ This observation suggested a novel mechanism to explain the stability of caspase- 2 in solution, although no mutational analysis was performed to support this hypothesis.

We have recently shown that endogenous procaspase-2 in cell lysates is spontaneously recruited to a large protein complex in a CARD-dependent manner, resulting in its activation. ${ }^{39}$ Our results also suggested that processing of the caspase-2 precursor is not required for its initial activation. ${ }^{39}$ In this study we have used recombinant, purified caspase- 2 proteins to delineate the biochemical mechanism of caspase- 2 activation. We demonstrate that initial activation of caspase-2 is achieved upon dimerization of caspase-2 zymogens, an event that does not depend upon disulfide bond linkage at the dimerization interface. Subsequent autocatalytic cleavage of caspase-2 occurs as a consequence of dimerization, resulting in stabilization of the dimer and optimal enzyme activation. Thus, caspase- 2 is shown to have a common mechanism of activation to caspase-8 and -9 , defining caspase-2 as a bona fide initiator caspase.

\section{Results}

\section{Cleavage of caspase-2 is not required for its initial activation}

In numerous published studies, the appearance of cleaved subunits of caspase-2 in apoptotic cell lysates is assumed to represent caspase-2 activation. However, we recently discovered using substrate-binding assays that activation of endogenous caspase-2 may occur without processing of the precursor molecule. ${ }^{39}$ To investigate the role of proteolytic cleavage in caspase-2 activation, we generated a number of recombinant mouse caspase- 2 proteins carrying cleavage site mutations. Caspase-2 precursor is known to undergo cleavage at D169, D333 and D347 to generate the fully processed enzyme (Figure 1a). ${ }^{13,31}$ When expressed in Escherichia coli, the protease domain of caspase-2 is first autoprocessed at D333, separating it into a large (p19) and a small (p14) subunit that remain associated and represent the catalytic portion of the molecule. ${ }^{13}$ Full maturation of caspase2 occurs when the prodomain is removed by cleavage at D169 and the intersubunit linker is removed by cleavage at D347, reducing the small subunit to $12 \mathrm{kDa}$ in size. ${ }^{12,31}$ For this reason, the recombinant proteins used in this study lack the prodomain and contain only the caspase-2 protease domain (residues 170-452) tagged with GST at the amino-terminus. Recombinant caspase-2 proteins were purified by affinity chromatography and their relative catalytic activities were determined by hydrolysis of the pentapeptide substrate, AcVDVAD-amc. Removal of GST or the use of $6 \times$ His tagged caspase-2 proteins gave results similar to those reported in this paper (data not shown), suggesting that GST fusion does not alter the biochemical properties of the caspase-2 proteins.

To investigate the role of cleavage in caspase-2 activation, we assessed the catalytic activity of an uncleavable mutant of caspase-2 (D333G), where the Asp333 residue has been mutated to Gly. Uncleavable D333G caspase-2 was found to retain up to $20 \%$ activity relative to the wild-type (WT) protein (Figure 1b), suggesting that cleavage of the protease

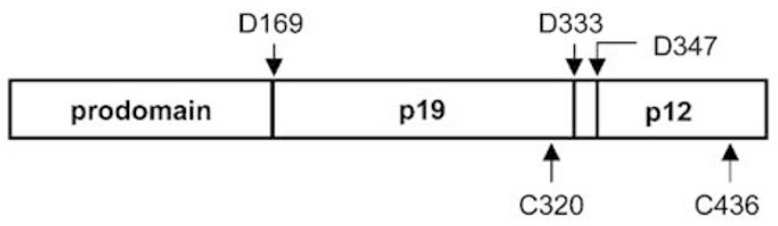

b

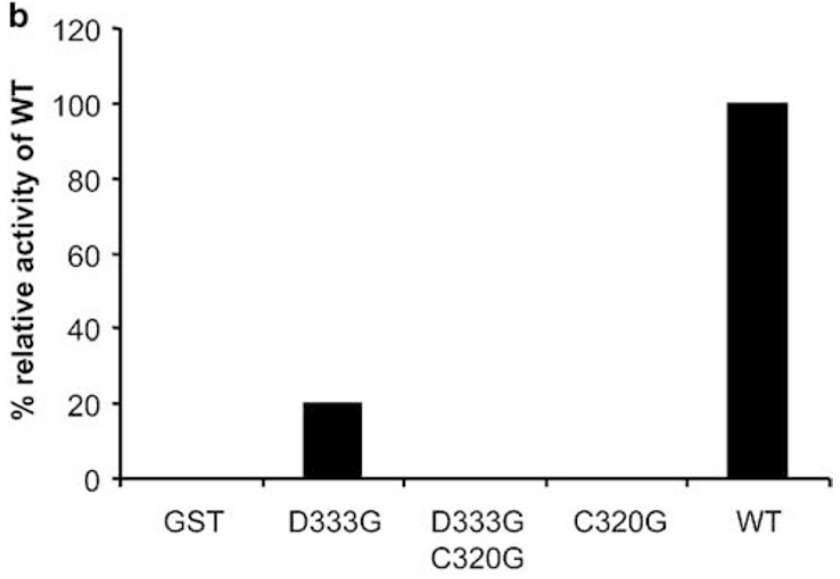

C

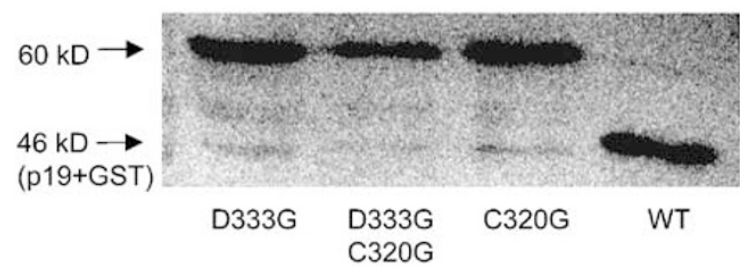

Figure 1 (a) Schematic representation of the caspase-2 protein. The mouse caspase-2 protein is 452 amino acids in length and consists of a long amino terminal prodomain and a carboxyl terminal protease domain (p19/p12). The Asp residues (D169, D333 and D347) at which processing occurs to generate the mature enzyme are indicated. The catalytic Cys (C320) and the Cys (C436) implicated in disulfide bond formation are also indicated. Purified recombinant proteins used in this study correspond to caspase-2 lacking the prodomain and contain a GST fusion at the amino terminus. (b) Processing of caspase-2 is not required for initial enzyme activity. Various recombinant GST-MPD-caspase-2 wild type and mutant proteins ( $5 \mathrm{nM}$ ) were tested for their relative catalytic activity by measuring hydrolysis of Ac-VDVAD-amc substrate after $1 \mathrm{~h}$ at $37^{\circ} \mathrm{C}$. This was determined to be in the linear range of caspase activity. (c) Lack of processing in mutant caspase-2 recombinant proteins. Recombinant caspase-2 proteins $(15 \mathrm{ng})$ were analyzed by electrophoresis and immunoblotting for evidence of processing between $\mathrm{p} 19$ and $\mathrm{p} 14$. The 11B4 antibody raised against the p19 subunit of caspase-2 detects the unprocessed GST-caspase-2 $(60 \mathrm{kDa})$ and GST-p19 (46 kDa) proteins

domain into two subunits is not an absolute requirement for initial caspase- 2 activation. In contrast, the catalytic activities of both the WT and the uncleavable D333G caspase-2 proteins were completely abolished by mutating the catalytic C320 residue (Figure 1b), even if tested at 100-fold higher concentrations (data not shown). The catalytic activity of the uncleavable caspase- 2 protein could not be ascribed to cleavage at alternative aspartate residue/s because the D333G mutant remained in its unprocessed form, confirming D333 as the primary site of cleavage in caspase-2 (Figure 1c). To determine whether the lower relative activity of the uncleavable mutant was due to a decreased affinity for the substrate or a lower catalytic efficiency, we determined the $K_{\mathrm{m}}$ 
Table 1 Catalytic activities of various recombinant caspase-2 proteins

\begin{tabular}{lccc}
\hline Protein & \multicolumn{1}{c}{$\boldsymbol{K}_{\mathbf{m}}(\boldsymbol{\mu M})$} & $\boldsymbol{K}_{\mathbf{c a t}}\left(\mathbf{s}^{-\mathbf{1}}\right)$ & $\boldsymbol{K}_{\text {cat }} / \boldsymbol{K}_{\mathbf{m}}\left(\mathbf{m}^{-\mathbf{1}} \mathbf{s}^{-\mathbf{1}}\right)$ \\
\hline WT & $33.99 \pm 8.6$ & 0.20 & $6.13 \times 10^{3}$ \\
D333G & $27.00 \pm 5.8$ & 0.04 & $1.57 \times 10^{3}$ \\
C436G & $33.13 \pm 6.73$ & 0.05 & $1.61 \times 10^{3}$ \\
\hline
\end{tabular}

and $K_{\text {cat }}$ values for both D333G and WT caspase-2 proteins (Table 1). While both proteins had similar affinity for the AcVDVAD-amc substrate, the rate of substrate cleavage was significantly lower in the uncleavable mutant. This infers that cleavage of caspase-2 results in conformational changes to the active site which further enhance the catalytic efficiency of the enzyme. Clearly, however, cleavage is not an essential requirement for the initial acquisition of enzyme activity. In summary, our biochemical analysis confirms that cleavage of caspase-2 is not required for initial enzyme activation, consistent with what has been reported recently for the initiator caspase- 8 and $-9^{4-7}$ and consistent with our previous data for caspase-2. ${ }^{39}$

\section{Cleavage of caspase-2 promotes its stable dimerization}

Recombinant mature caspase-2 exists almost exclusively as a dimer in solution. ${ }^{38}$ We hypothesized that proteolytic cleavage may be important for stable dimerization of caspase-2. To assess whether uncleavable caspase-2 can form stable dimers in solution, we performed gel filtration analysis and compared the elution profiles of WT caspase-2 and uncleavable D333G caspase-2 proteins by immunoblotting. Consistent with previously published work, elution of WT caspase-2 peaks in fractions 15-18 corresponding to the expected size of a GST-caspase-2 dimer ( $120 \mathrm{kDa})$ (Figure 2a). Strikingly, uncleavable caspase-2 predominantly elutes in fractions 19-22 corresponding to the estimated size of a GST-caspase-2 monomer (60 kDa) (Figure 2b). Thus, introduction of a single mutation that prevents intersubunit cleavage results in an inability of caspase-2 to form stable dimers in solution. We conclude therefore that proteolytic cleavage of caspase-2 at D333 is associated with its stable dimerization.

\section{Dimerization is required for caspase-2 activation}

To assess whether the intrinsic activity of the uncleavable D333G protein is attributable to a monomeric protein species, we assayed each protein fraction collected from the gel filtration column for the ability to hydrolyze the Ac-VDVADamc substrate. A small but detectable level of D333G activity was observed to peak in fractions 15-18, despite the fact that the vast majority of D333G protein clearly elutes as a monomeric species (Figure 2b). WT enzyme activity also peaked in fractions 15-18, coincident with the peak of dimeric protein (Figure 2a). As fractions 15-18 are expected to contain dimeric protein species, we attribute the intrinsic catalytic activity of the D333G protein measured in these
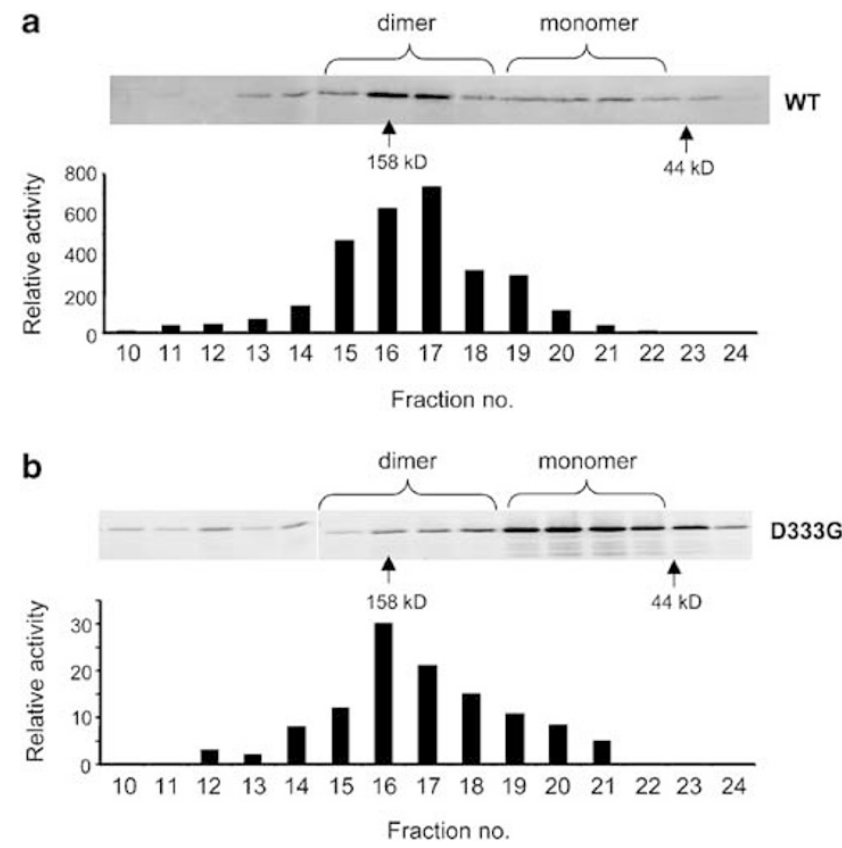

Figure 2 Processing is required for stable dimerization of caspase-2 and activity is associated with the dimeric species. (a) Wild-type caspase-2 protein ( $150 \mathrm{pmol}$ ) was subjected to gel filtration using a Superdex 200 column. Western blot for caspase-2 of the collected fractions indicates the majority of the protein eluted as a dimer $(\sim 120 \mathrm{kDa})$. Catalytic activity against Ac-VDVAD-amc is also associated with the fractions corresponding to dimeric protein. (b) Mutant D333G caspase-2 $(150 \mathrm{pmol})$ was subjected to gel filtration using a Superdex 200 column and the fractions assayed for caspase-2 by Western blot. Majority of the protein eluted as a monomer $(\sim 60 \mathrm{kDa})$. Activity against Ac-VDVAD-amc was detected in fractions associated with dimeric protein. Note the difference in relative activity between wild type and mutant D333G caspase-2

fractions to transient dimer formation between single-chain zymogens. Taken together, our data strongly suggest that dimerization (and not cleavage) is the key event required for initial caspase-2 activation.

\section{Disulfide bond formation between caspase-2 monomers is dispensable for stable dimerization of caspase-2}

Under crystallization conditions, two caspase-2 monomers are covalently linked at the dimerization interface by a central disulfide bridge. ${ }^{38}$. To assess the importance of this disulfide bond for stable dimerization of caspase-2, we generated recombinant GST-caspase-2 containing a Cys to Gly substitution of residue 436 involved in disulfide bond formation. Surprisingly, caspase-2 C436G eluted as a dimeric species by gel filtration analysis and was fully processed in $E$. coli in identical manner to the WT protein (Figure 3). We also noted the formation of some higher molecular mass complexes of caspase-2 C436G (data not shown), but the reason for this is currently not known. Thus, prevention of disulfide bond formation at the dimerization interface did not affect the ability of recombinant caspase-2 to dimerize and undergo autoprocessing. To assess whether mutation of $\mathrm{C} 436$ could affect the catalytic efficiency of the enzyme, we determined the $K_{\text {cat }}$ value of the C436G mutant and found it to be approximately $25 \%$ that of WT enzyme while the $K_{m}$ value was similar to WT 


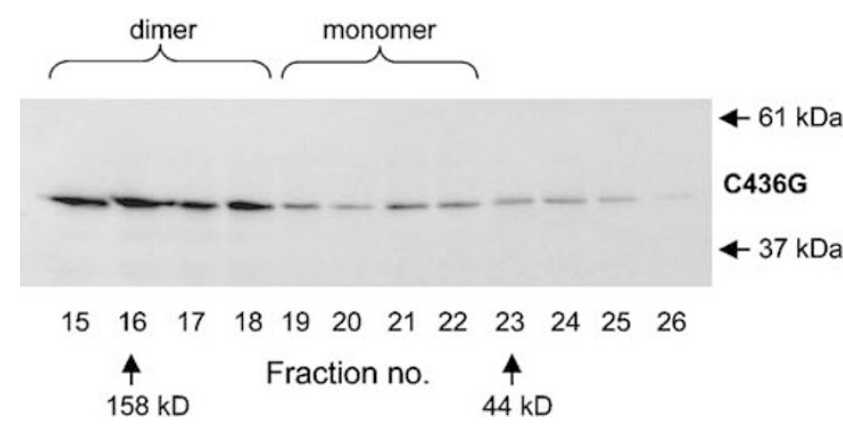

Figure 3 Disulfide bond formation at the dimerization interface is dispensable for caspase-2 dimerization in vitro. C436G caspase-2 protein (150 pmol) was subjected to gel filtration using a Superdex 200 column. Western blot for caspase- 2 of the collected fractions indicates the majority of the protein eluted as a dimer $(\sim 120 \mathrm{kDa})$ or larger and not as a monomer. Expression and purification of C436G caspase-2 protein yields protein of $46 \mathrm{kDa}$ size, indicative of autocatalytic cleavage in bacteria. Some higher molecular mass complexes (likely tetramers) were also seen with the C436G caspase-2 protein (data not shown)

(Table 1). This indicates that while mutation of C436G does not negatively impact on the ability of caspase-2 to dimerize, it does appear to reduce the catalytic efficiency of the caspase2 enzyme by an unknown mechanism. We conclude therefore that disulfide bond linkage between two caspase-2 monomers is dispensable for stable dimerization of caspase-2 in vitro.

\section{Both dimerization and cleavage of caspase-2 are required for cell death induced by caspase-2 overexpression}

To test the physiological relevance of our biochemical data, we cloned the full-length versions of the uncleavable D333G and the disulfide bond C436G caspase-2 mutants into mammalian expression vectors and expressed them in HEK-293T cells. The ability of the caspase- 2 mutants to induce apoptosis in transfected cells was assessed by scoring transfected cells for apoptotic morphology and compared to WT or appropriate negative controls. Despite the fact that cleavage is not required for initial caspase- 2 activation, the full-length D333G caspase-2 mutant was unable to induce significant levels of apoptosis in transfected cells (Figure 4a). This may be due to the relatively lower catalytic activity of D333G. Recently published results indicate that PIDDmediated activation of a substantial pool of endogenous caspase-2 is insufficient for killing cells. Therefore, it is possible that the rapid induction of apoptosis induced by overexpressed caspase- 2 requires fully active dimeric enzyme, which may be achieved by cleavage at D333. Thus, when apoptosis is induced by overexpression of caspase-2, cleavage of caspase-2 appears to be critical for its biological effect. This result confirms our findings in a previous study. ${ }^{13}$ Consistent with the biochemical data, the C436G mutant retained the ability to kill transfected cells, albeit with lower efficiency when compared to the WT caspase-2 (Figure 4a). As expected, the catalytically inactive C320G mutant did not induce cell death.

Western blot analysis of transfected cell lysates demonstrated cleavage of WT and C436G proteins between the large and small subunits, as detected by the p14/p12-GFP

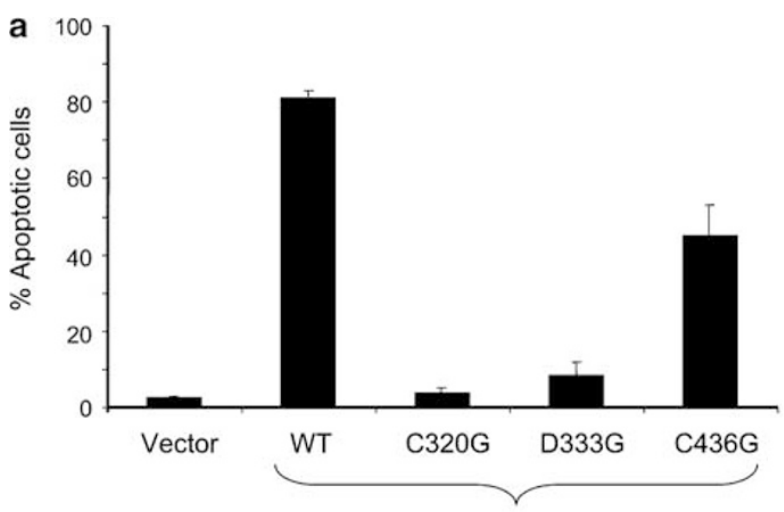

Caspase-2 expression constructs

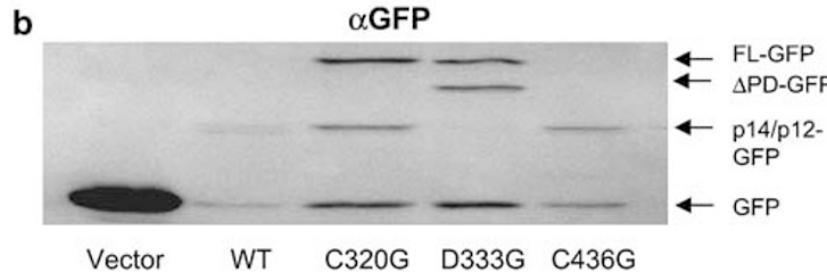

Figure 4 Effect of mutations on cell killing induced by caspase-2 overexpression. (a) HEK-293T cells were cotransfected with the indicated caspase-2 constructs and a $\beta$-galactosidase expression vector. Following $24 \mathrm{~h}$ after transfection, cells were fixed and stained with X-gal and $\beta$-galactosidase positive cells were scored for apoptotic morphology by microscopic examination. Data ( \pm S.E.M.) were derived from a single experiment. (b) Immunoblotting with GFP antibody reveals the presence of processed products of caspase-2-GFP fusion proteins in transfected HEK-293T cell lysates. FL, full length; $\triangle \mathrm{PD}$, lacking the prodomain

fragment (Figure 4b). Complete absence of the full-length precursor and the appearance of the small subunit correlates well with the ability of WT and C436G proteins to kill transfected cells (Figure 4a). In contrast, the D333G protein is prevented from being cleaved at this site and has a correspondingly poor ability to induce cell death. Interestingly, some of the D333G protein undergoes proteolytic cleavage at D169, releasing the protease domain fused to GFP (59 kDa) from the $\mathrm{N}$-terminal prodomain (Figure $4 \mathrm{~b}$ ). Some processing also appears to occur in cells transfected with the catalytically inactive C320G mutant, a curious observation we have noted previously. ${ }^{13,40}$ In light of our findings described below, cleavage of the C320G protein could be explained by an ability to dimerize with endogenous WT caspase-2, though such an event does not lead to an increase in apoptosis. In summary, our results suggest that while cleavage of caspase2 is not required for initial activation, it is critical for its ability to kill cells. This is consistent with our findings that cleavage of caspase- 2 results in its stable dimerization, which in turn increases the catalytic efficiency of the enzyme.

\section{Dimerization can induce caspase-2 cleavage via an intramolecular mechanism}

Finally, to investigate how each caspase-2 zymogen becomes catalytically competent following dimerization we designed an in vitro dimerization assay. Lysates from $E$. coli cells expressing various full-length GST-caspase-2 proteins were 
a
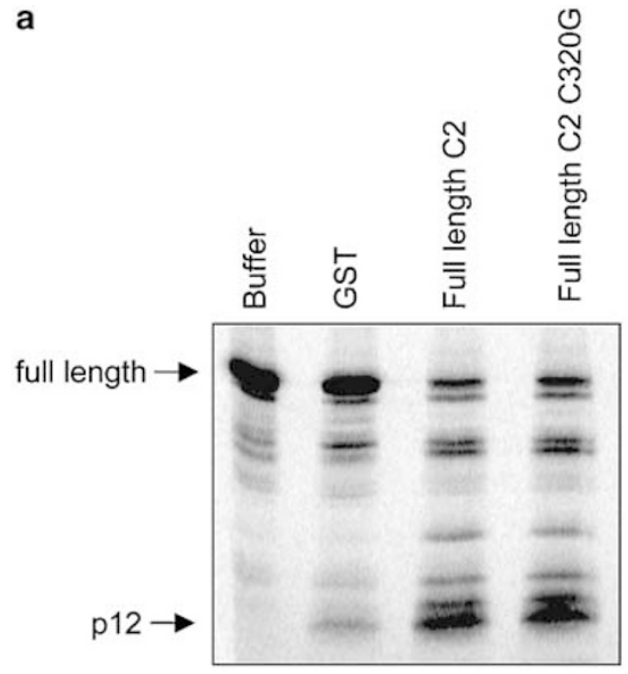

IVT

procaspase-2

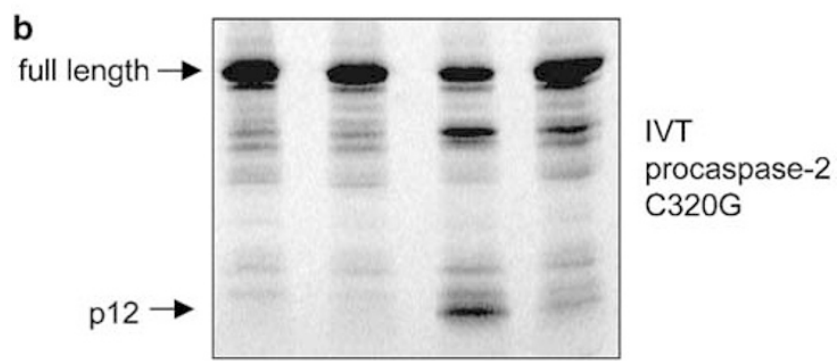

Figure 5 Dimerization induces processing of caspase-2 by an intramolecular mechanism. $\left[{ }^{35} \mathrm{~S}\right]$ methionine-labelled IVT wild type (a) and catalytically inactive (C320G) (b) caspase-2 proteins were incubated with various recombinant GST caspase-2 proteins for $3 \mathrm{~h}$ at $37^{\circ} \mathrm{C}$. Processing of the IVT protein was detected by electrophoresis and phosphorimaging. C2, caspase-2

co-incubated with in vitro translated (IVT), ${ }^{35} \mathrm{~S}$-methioninelabelled procaspase-2 proteins (WT and C320G). Dimerization between the co-incubated proteins was allowed to occur at $37^{\circ} \mathrm{C}$, then the processed products of the IVT protein were assessed by SDS-PAGE and phosphorimaging. It was necessary to use lysates in this experiment as purification of full-length caspase-2 is technically difficult because of autocatalytic cleavage between the large subunit and the prodomain in bacteria. Addition of buffer alone or lysates from E. coli expressing GST does not result in any significant cleavage of IVT procaspase-2 (Figure $5 a$, lanes 1 and 2 ) or IVT procaspase-2 C320G (Figure 5b, lanes 1 and 2). This is presumably because the IVT protein is not at a high enough concentration for spontaneous dimerization of caspase-2 to occur, as it does in E. coli. As expected, addition of full-length WT caspase-2 results in the cleavage of IVT procaspase-2 (Figure 5a, lane 3 ) and IVT procaspase-2 C320G (Figure 5b, lane 3). Surprisingly however, the addition of full-length C320G caspase-2, which lacks a functional active site, could also promote cleavage of IVT procaspase-2 (Figure $5 \mathrm{a}$, lane 4). In contrast, the catalytically inactive $C 320 \mathrm{G}$ protein was unable to promote the cleavage of IVT procaspase-2 C320G (Figure $5 \mathrm{~b}$, lane 4). Our data suggest that upon forming a dimer with full-length caspase-2 C320G protein, the IVT procaspase-2 monomer undergoes self-cleavage, as this does not occur when the IVT protein is catalytically inactive. Taken together, our results indicate that upon dimerization of two procaspase-2 molecules, subsequent intersubunit cleavage at D333 can occur by an intramolecular mechanism.

\section{Discussion}

Using a biochemical approach, we have provided data to explain how the single-chain caspase-2 zymogen can achieve catalytic competence. Importantly, we demonstrate that caspase-2 does not require processing for its initial activation, consistent with recent evidence that it functions as an initiator caspase during apoptosis. ${ }^{20-28}$ Instead, dimerization between the protease domains of the two caspase-2 monomers is the key event required for initial caspase-2 zymogen activation. Subsequent cleavage at D333 promotes stable dimerization of caspase-2 and further enhances the catalytic efficiency of the enzyme. Our data also suggest that disulfide bond linkage at the dimerization interface is unlikely to contribute significantly to the stable dimerization of caspase-2 in solution. Based on our findings in this study, we propose a model for procaspase-2 activation (Figure 6).

Caspase-2 contains a CARD, a conserved motif only found in initiator caspases in C. elegans, Drosophila and mammals. ${ }^{1,2,41,42}$ The ability of caspase-2 to autoactivate by a dimerization-dependent mechanism is also consistent with it being an initiator caspase. Our findings for caspase-2 are in good agreement with recent studies focusing on the biochemical mechanisms of initiator caspase activation. ${ }^{4-7}$ Like caspase-2, caspase-8 is initially activated by dimerization and processing occurs as a secondary event, which serves to stabilize the newly formed dimer. ${ }^{5,7}$ Artificially-induced dimerization of caspase-8, -9 and -10 also results in enzyme activation by an intramolecular mechanism, ${ }^{4}$ consistent with our findings for caspase-2 in this study. Mutation of the C436 residue implicated in disulfide bond formation at the caspase2 dimerization interface, ${ }^{38}$ had no apparent effect on the ability of caspase-2 to dimerize in vitro. However, we cannot rule out the possibility that redox conditions in vivo may modulate activation of caspase-2 by dimerization. The reduction in catalytic efficiency observed for this mutant may also be due to structural/conformational changes in the active site loop.

As the first cleavage can occur by self-cleavage within each monomer, only one active site may need to be functional in order to generate proteolytic activity. In fact, caspase-9 has been shown to possess only one functional active site in its dimeric form. ${ }^{2,3}$ This has consequences when using catalytically inactive caspase-2 mutants as negative controls or dominant-negative molecules as they could potentially bind and activate endogenous caspase-2. Indeed, we have observed in this study and previously ${ }^{13,40}$ a small amount of the p14 subunit in cell lysates transfected with the caspase-2 C320G mutant, perhaps a direct result of endogenous caspase-2 activation. Similarly, a proportion of the protease domain of the D333G protein was liberated by cleavage at the prodomain-p19 cleavage site. Although these mutants have the capacity to bind and promote self-cleavage of endogenous caspase-2, because they are expressed in such large excess they would be predicted to out-compete endogenous caspase-2 for its substrate/s, preventing induction of cell death 


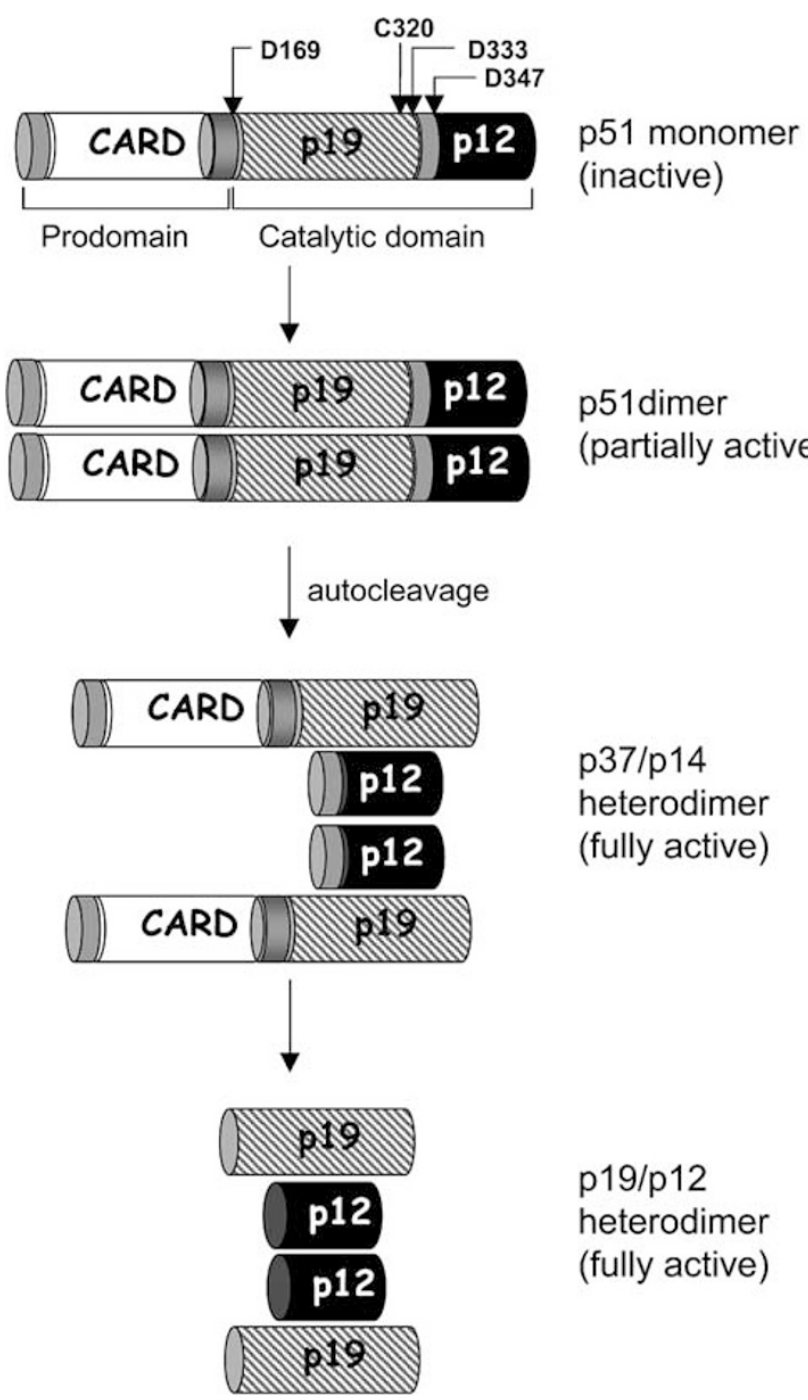

Figure 6 A model for the mechanism of caspase-2 activation in vivo. We propose that procaspase-2 molecules exist as monomers at normal physiological concentrations. In response to a proapoptotic signal, procaspase-2 molecules are recruited into close proximity via interactions mediated through the prodomain and an adaptor molecule such as RAIDD. If present in high enough concentrations, such as in overexpressing cells or when purified from $E$. coli, caspase-2 molecules can aggregate spontaneously via interactions between the prodomains, the protease domains or both. When brought into close proximity, the protease domains of caspase-2 can interact to form a transient dimeric complex, which is sufficient to impart some intrinsic catalytic activity to the unprocessed zymogens. Dimerization is followed by cleavage between the large and small subunits (at D333) of the protease domains of each participating monomer, most likely by an intramolecular mechanism. Intramolecular cleavage leads to the formation of an active stable tetrameric complex, which attains maximal catalytic competence. Disulfide bond formation at the dimerization interface is unlikely to contribute to the stable dimerization of caspase-2. Further processing, possibly by caspase- 3 , generates the fully mature $(p 19+p 12)_{2}$ caspase-2 enzyme. In the three-dimensional structure, the two caspase-2 monomers will be oriented in an antiparallel manner. For simplicity, this is not depicted in this model

by endogenous caspase-2. This is consistent with the lack of toxicity caused by these mutants.

Previous studies, including some from our own laboratory, have shown that most of the detectable caspase-2 cleavage in dying cells appears to be mediated by active caspase- 3 .
Yet, our biochemical analysis has shown that initial activation of caspase-2 occurs by dimerization and not by cleavage per sé. As cleavage at D333 to generate fully active caspase-2 can be mediated by the autocatalytic activity of caspase-2, the significance of caspase-3-mediated cleavage of caspase-2 remains to be established. In this context, it is interesting that in some cell types deficient in either caspase- 9 or its adaptor, Apaf-1, apoptosis still occurs with little detectable caspase-3 cleavage. ${ }^{43,44}$ Thus in these cells, only a small amount of active caspase may be sufficient to initiate and mediate cell death. This raises the possibility that the majority of caspase cleavage normally seen in apoptotic cell lysates is the result of a positive amplification loop operating via mitochondria, which is deficient in these cells.

Caspase-2 self-associates readily at high concentrations but at endogenous levels, caspase-2 would presumably require an adaptor to bring caspase-2 zymogens into close proximity to allow dimerization, the key event required for its initial activation. Supporting this idea, a recent study shows that RAIDD can bind to and promote caspase- 2 activation by recruiting it into a protein complex that also contains PIDD, a p53-inducible death domain-containing protein. ${ }^{20}$

While elucidation of the mechanism of initial caspase-2 activation is important for understanding its function, the identity of its downstream targets remain to be discovered. Recombinant caspase-2 is able to cause cytochrome $c$ release from isolated mitochondria in vitro, ${ }^{27}$ suggesting that the downstream target of activated caspase-2 could be a mitochondrial protein. On the other hand, localization of caspase-2 in the nucleus ${ }^{16,28}$ suggests that it is likely to cleave nuclear substrate(s), particularly in response to genotoxic stress signals. In future studies, identification of the potential downstream targets of caspase-2 will be necessary to fully delineate the physiological function of this caspase.

\section{Materials and Methods}

\section{Cell culture}

HEK293T cells were maintained in Dulbecco's modified Eagle's medium supplemented with $10 \%$ fetal bovine serum, $100 \mathrm{U} / \mathrm{ml}$ penicillin, $100 \mu \mathrm{g} / \mathrm{ml}$ streptomycin and $2 \mathrm{mM} \mathrm{L-glutamine.}$

\section{Bacterial and mammalian expression constructs}

The coding region of mouse caspase-2 lacking the prodomain (residues 170-452) was amplified by PCR and fused in-frame to the carboxyl terminus of GST in the expression vector, pGEX4T3 (Amersham Biosciences) using BamH1 and Xho1 restriction sites. Single amino-acid mutations (C320G, D333G and C436G) were created by PCR-based mutagenesis. In the case of full-length proteins, mouse caspase-2 coding region (residues 22-452) was fused in-frame to GST using BamH1 restriction sites in the pGEX4T3 expression vector and mutants created as above. WT and catalytically inactive mouse caspase-2-GFP constructs have been described previously. ${ }^{40}$ WT construct was used as a template for PCR-based mutagenesis to introduce the D333G and C436G mutations. WT and catalytically inactive mouse caspase-2 cDNAs were cloned into the BamH1 and Xba1 sites of the pcDNA3.1 expression vector (Invitrogen) with a carboxyl-terminal Flag tag and used as templates for the production of $\left[{ }^{35} \mathrm{~S}\right]$ methionine-labelled proteins. 


\section{Purification of GST fusion proteins}

GST fusion proteins were purified using glutathione Sepharose beads, and eluted using glutathione elution buffer according to the manufacturer's protocol. The concentration of purified protein was determined by dyebinding assay (Biorad) and the extent of purity was determined by SDSPAGE of the purified proteins followed by Sypro Orange staining (Molecular Probes) (1/5000 in 7.5\% acetic acid). The gel was scanned and analyzed using Typhoon 9410 (Amersham Biosciences) and ImageQuant software (Molecular Dynamics).

\section{Caspase activity assay}

Purified GST-fusion proteins were diluted in assay buffer (25 mM HEPES $\mathrm{pH} 7.4,10 \%$ sucrose, $0.1 \%$ CHAPS, $1 \mathrm{mM}$ EDTA, $10 \mathrm{mM}$ DTT) containing $100 \mu \mathrm{M} \quad$ Acetyl-Val-Asp-Val-Ala-Asp-Amino-methylcoumaride (AcVDVAD-amc) substrate (California Peptide Research) in a final volume of $50 \mu \mathrm{l}$. Peptide hydrolysis was allowed to occur for $60 \mathrm{~min}$ at $37^{\circ} \mathrm{C}$, and then VDVAD-amc cleavage was measured at excitation $385 \mathrm{~nm}$ and emission $460 \mathrm{~nm}$ wavelengths on a luminescence spectrometer (PerkinElmer, LS-55). For kinetic studies, enzyme assays were carried out at room temperature $\left(\sim 22^{\circ} \mathrm{C}\right)$. The enzyme kinetics were analyzed over the substrate concentration range of $3.125-200 \mu \mathrm{M}$ using Michaelis-Menten kinetics with the nonlinear regression program, Hyper $1.01 \mathrm{~s}$. We observed a concentration-dependent effect on the relative catalytic activities between different batches of purified proteins. For example, the D333G protein exhibited relative catalytic activities of between 1 and $25 \%$ depending on the batch of protein used.

\section{Chromatography}

Purified protein was fractionated using an FPLC protein purification system on a Superdex 200 column (Amersham Biosciences) at $4^{\circ} \mathrm{C}$. The column was equilibrated with buffer ( $20 \mathrm{mM}$ Tris- $\mathrm{Cl}, \mathrm{pH} 7.5,100 \mathrm{mM} \mathrm{NaCl}$, $5 \%$ glycerol) and protein $(150 \mathrm{pmol})$ was applied and eluted from the column with buffer at a flow rate of $0.4 \mathrm{ml} / \mathrm{min}$ and $400 \mu$ l fractions were collected. The column was calibrated with Bio-Rad Laboratories gel filtration standards containing bovine thyroglobulin $(670 \mathrm{kDa})$, horse gamma globulin $(158 \mathrm{kDa})$, chicken ovalbumin $(44 \mathrm{kDa})$, horse myoglobin $(17 \mathrm{kDa})$ and vitamin $\mathrm{B}_{12}(1.35 \mathrm{kDa})$.

\section{SDS-PAGE electrophoresis and immunoblotting}

Purified GST-caspase-2 fusion proteins ( $15 \mathrm{ng}$ ), fractions collected from gel filtration analysis $(30 \mu \mathrm{l})$ or lysates from transfected HEK-293T cells were resolved on $12 \%$ polyacrylamide gels and transferred onto polyvinylidine difluoride (PVDF) membrane (Hybond-P, Amersham Biosciences). Membranes were blocked in $5 \%$ nonfat milk and incubated with monoclonal 11B4 caspase-2 antibody ${ }^{34}$ or anti-GFP monoclonal antibody (Roche). Antibody binding was detected using goat anti-rat IgG conjugated to alkaline phosphatase, or sheep anti-mouse IgG conjugated to alkaline phosphatase (Pierce), respectively, and ECF (Amersham Biosciences) using the Typhoon 9410 and ImageQuant software.

\section{Apoptosis assay}

For cell death assays, $10^{5}$ cells were seeded into $35 \mathrm{~mm}$ dishes and transfected $48 \mathrm{~h}$ later with a total of $2 \mu \mathrm{g}$ of DNA (1.6 $\mu \mathrm{g}$. of caspase-2 constructs and $0.4 \mu \mathrm{g}$ of $\mathrm{pEF}-\beta \mathrm{gal}$ ) using FuGENE6 transfection reagent (Roche Biochemicals), according to the manufacturer's protocol. After
$24 \mathrm{~h}$, cells were fixed in $2 \%$ formaldehyde $/ 0.2 \%$ glutaraldehyde in PBS and stained with X-gal as described previously. ${ }^{9,45}$ Transfected cells ( $\beta$ galactosidase positive) were scored for apoptotic morphology by microscopic examination.

\section{In vitro translation}

A coupled transcription/translation kit (Promega) was used to produce ${ }^{35}$ S]methionine-labelled proteins according to the manufacturer's protocol. Translated caspase-2 product $(3 \mu \mathrm{l})$ was incubated with lysate from E. coli expressing recombinant GST-caspase-2 fusion proteins, for $3 \mathrm{~h}$ at $37^{\circ} \mathrm{C}$. Samples were resolved on a $15 \%$ SDS-PAGE gel, transferred to PVDF and exposed to a phosphor screen for $48 \mathrm{~h}$. Cleavage of IVT caspase-2 protein was assessed by phosphorimaging using the Typhoon 9410 and ImageQuant software.

\section{Acknowledgements}

We thank members of our laboratory and Stuart Pitson for the supply of reagents and helpful discussions. This work was supported by the National Health and Medical Research Council of Australia, and the Cancer Council of South Australia.

\section{References}

1. Kumar S and Colussi PA (1999) Prodomains-adaptors-oligomerization: the pursuit of caspase activation in apoptosis. Trends Biochem. Sci. 24: 1-4

2. Shi $Y$ (2002) Mechanisms of caspase activation and inhibition during apoptosis. Mol. Cell 9: 459-470

3. Salvesen GS and Abrams JM (2004) Caspase activation-stepping on the gas or releasing the brakes? Lessons from humans and flies. Oncogene 23 : 2772-2784

4. Chen M, Orozco A, Spencer DM and Wang J (2002) Activation of initiator caspases through a stable dimeric intermediate. J. Biol. Chem. 277: 50761-50767

5. Donepudi M, MacSweeney A, Briand C and Grutter MG (2003) Insights into the regulatory mechanism for caspase-8 activation. Mol. Cell 11: 543-549

6. Chang DW, Ditsworth D, Liu H, Srinivasula SM, Alnemri ES and Yang X (2003) Oligomerization is a general mechanism for the activation of apoptosis initiator and inflammatory procaspases. J. Biol. Chem. 278: 16466-16469

7. Boatright KM, Renatus M, Scott FL, Sperandio S, Shin H, Pedersen IM, Ricci JE, Edris WA, Sutherlin DP, Green DR and Salvesen GS (2003) A unified model for apical caspase activation. Mol. Cell 11: 529-541

8. Fischer U, Janicke RU and Schulze-Osthoff K (2003) Many cuts to ruin: a comprehensive update of caspase substrates. Cell Death Differ. 10: 76-100

9. Kumar S, Kinoshita M, Noda M, Copeland NG and Jenkins NA (1994) Induction of apoptosis by the mouse Nedd2 gene which encodes a protein similar to the product of the Caenorhabditis elegans cell death gene ced-3 and the mammalian IL-1 beta-converting enzyme. Genes Dev. 8: 1613-1626

10. Wang L, Miura M, Bergeron L, Zhu H and Yuan J (1994) Ich-1 an Ice/ced-3related gene encodes both positive and negative regulators of programmed cell death. Cell 78: 739-750

11. Kumar S, Kinoshita M, Dorstyn L and Noda M (1997) Origin expression and possible functions of the two alternatively spliced forms of the mouse Nedd2 mRNA. Cell Death Differ. 4: 378-387

12. Harvey NL, Butt AJ and Kumar S (1997) Functional activation of Nedd2/ICH-1 (caspase-2) is an early process in apoptosis. J. Biol. Chem. 272: 13134-13139

13. Butt AJ, Harvey NL, Parasivam G and Kumar S (1998) Dimerization and autoprocessing of the Nedd2 (caspase-2) precursor requires both the prodomain and the carboxyl-terminal regions. J. Biol. Chem. 273: 6763-6768

14. Colussi PA, Harvey NL, Shearwin-Whyatt LM and Kumar S (1998) Conversion of procaspase-3 to an autoactivating caspase by fusion to the caspase-2 prodomain. J. Biol. Chem. 273: 26566-26570 
15. Shearwin-Whyatt L, Baliga B, Doumanis J and Kumar S (2001) Chimeric caspase molecules with potent cell killing activity in apoptosis-resistant cells. Biochem. Biophys. Res. Commun. 282: 1114-1119

16. Baliga BC, Colussi PA, Read SH, Dias MM, Jans DA and Kumar S (2003) Role of prodomain in importin-mediated nuclear localization and activation of caspase-2. J. Biol. Chem. 278: 4899-4905

17. Duan H and Dixit VM (1997) RAIDD is a new 'death' adaptor molecule. Nature 385: $86-89$

18. Ahmad M, Srinivasula SM, Wang L, Talanian RV, Litwack G, FernandesAlnemri T and Alnemri ES (1997) CRADD a novel human apoptotic adaptor molecule for caspase-2 and FasL/tumor necrosis factor receptor-interacting protein RIP. Cancer Res. 57: 615-619

19. Shearwin-Whyatt LM, Harvey NL and Kumar S (2000) Subcellular localization and CARD-dependent oligomerization of the death adaptor RAIDD. Cell Death Differ. 7: 155-165

20. Tinel A and Tschopp J (2004) The PIDDosome, a protein complex implicated in activation of caspase-2 in response to genotoxic stress. Science 304: 843-846

21. Lassus $P$, Opitz-Araya $X$ and Lazebnik $Y$ (2002) Requirement for caspase-2 in stress-induced apoptosis before mitochondrial permeabilization. Science 297: 1352-1354

22. Jiang M and Milner J (2003) Bcl-2 constitutively suppresses p53-dependent apoptosis in colorectal cancer cells. Genes Dev. 17: 832-837

23. Castedo M, Perfettini JL, Roumier T, Valent A, Raslova H, Yakushijin K, Horne D, Feunteun J, Lenoir G, Medema R, Vainchenker W and Kroemer G (2004) Mitotic catastrophe constitutes a special case of apoptosis whose suppression entails aneuploidy. Oncogene 23: 4362-4370

24. Li D, Das S, Yamada T and Samuels HH (2004) The NRIF3 family of transcriptional coregulators induces rapid and profound apoptosis in breast cancer cells. Mol. Cell. Biol. 24: 3838-3848

25. Kumar S (1995) Inhibition of apoptosis by the expression of antisense Nedd2. FEBS Lett. 368: 69-72

26. Robertson JD, Enoksson M, Suomela M, Zhivotovsky B and Orrenius S (2002) Caspase-2 acts upstream of mitochondria to promote cytochrome $c$ release during etoposide-induced apoptosis. J. Biol. Chem. 277: 29803-29809

27. Guo Y, Srinivasula SM, Druilhe A, Fernandes-Alnemri T and Alnemri ES (2002) Caspase-2 induces apoptosis by releasing proapoptotic proteins from mitochondria. J. Biol. Chem. 277: 13430-13437

28. Paroni G, Henderson C, Schneider $C$ and Brancolini $C$ (2002) Caspase-2 can trigger cytochrome $C$ release and apoptosis from the nucleus. J. Biol. Chem. 277: $15147-15161$

29. Kumar S and Vaux DL (2002) Apoptosis. A cinderella caspase takes center stage. Science 297: 1290-1291

30. Baliga B and Kumar S (2003) Apaf-1/cytochrome $c$ apoptosome: an essential initiator of caspase activation or just a sideshow? Cell Death Differ. 10: $16-18$

31. Harvey NL, Trapani JA, Fernandes-Alnemri T, Litwack G, Alnemri ES and Kumar S (1996) Processing of the Nedd2 precursor by ICE-like proteases and granzyme B. Genes Cells 1: 673-685
32. Paroni G, Henderson C, Schneider C and Brancolini C (2001) Caspase-2induced apoptosis is dependent on caspase-9 but its processing during UV- or tumor necrosis factor-dependent cell death requires caspase-3. J. Biol. Chem. 276: $21907-21915$

33. Slee EA, Harte M, T Kluck RM, Wolf BB, Casiano CA, Newmeyer DD, Wang HG, Reed JC, Nicholson DW, Alnemri ES, Green DR and Martin SJ (1999) Ordering the cytochrome $c$-initiated caspase cascade: hierarchical activation of caspases-2 -3 -6 -7 -8 and -10 in a caspase-9-dependent manner. J. Cell Biol. 144: $281-292$

34. O'Reilly LA, Ekert P, Harvey N, Marsden V, Cullen L, Vaux DL, Hacker G, Magnusson C, Pakusch M, Cecconi F, Kuida K, Strasser A, Huang DC and Kumar S (2002) Caspase-2 is not required for thymocyte or neuronal apoptosis even though cleavage of caspase-2 is dependent on both Apaf-1 and caspase9. Cell Death Differ. 9: 832-841

35. Troy CM and Shelanski ML (2003) Caspase-2 redux. Cell Death Differ. 10: 101-107

36. Creagh EM and Martin SJ (2003) Cell stress-associated caspase activation: intrinsically complex? Sci. STKE 175: pe11

37. Bergeron L, Perez GI, Macdonald G, Shi L, Sun Y, Jurisicova A, Varmuza S, Latham KE, Flaws JA, Salter JC, Hara H, Moskowitz MA, Li E, Greenberg A, Tilly JL and Yuan J (1998) Defects in regulation of apoptosis in caspase-2deficient mice. Genes Dev. 12: 1304-1314

38. Schweizer A, Briand C and Grütter MG (2003) Crystal structure of caspase-2 apical initiator of the intrinsic apoptotic pathway. J. Biol. Chem. 278: 42441-42447

39. Read SH, Baliga BC, Ekert PG, Vaux DL and Kumar S (2002) A novel Apaf-1independent putative caspase-2 activation complex. J. Cell Biol. 159: 739-745

40. Colussi PA, Harvey NL and Kumar S (1998) Prodomain-dependent nuclear localization of the caspase-2 (Nedd2) precursor A novel function for a caspase prodomain. J. Biol. Chem. 273: 24535-24542

41. Lamkanfi M, Declercq W, Kalai M, Saelens X and Vandenabeele P (2002) Alice in caspase land. A phylogenetic analysis of caspases from worm to man. Cell Death Differ. 9: 358-361

42. Dorstyn L, Colussi PA, Quinn LM, Richardson H and Kumar S (1999) DRONC an ecdysone-inducible Drosophila caspase. Proc. Natl. Acad. Sci. USA 96: 4307-4312

43. Marsden VS, O'Connor L, O'Reilly LA, Silke J, Metcalf D, Ekert PG, Huang DC, Cecconi F, Kuida K, Tomaselli KJ, Roy S, Nicholson DW, Vaux DL, Bouillet P, Adams JM and Strasser A (2002) Apoptosis initiated by Bcl-2-regulated caspase activation independently of the cytochrome c/Apaf- $1 /$ caspase- 9 apoptosome. Nature 419: 634-637

44. Ekert PG, Read SH, Silke J, Marsden VS, Kaufmann H, Hawkins CJ, Gerl R, Kumar S and Vaux DL (2004) Apaf-1 and caspase-9 accelerate apoptosis, but do not determine whether factor-deprived or drug-treated cells die. J Cell Biol. 165: 835-842

45. Dorstyn L and Kumar S (1997) Differential inhibitory effects of CrmA P35 IAP and three mammalian IAP homologues on apoptosis in NIH3T3 cells following various death stimuli. Cell Death Differ. 4: 570-579 